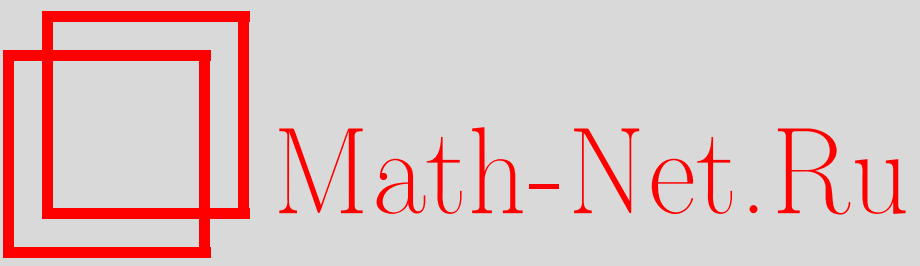

Л. Ю. Кабанцова, Об условиях обратимости разностных операторов, Итоги науки и техн. Сер. Соврем. мат. и ее прил. Темат. обз., 2019, том 171, 94-101

DOI: https://doi.org/10.36535/0233-6723-2019-171-94-101

Использование Общероссийского математического портала Math-Net.Ru подразумевает, что вы прочитали и согласны с пользовательским соглашением

http://www.mathnet.ru/rus/agreement

Параметры загрузки:

IP: 54.80 .97 .219

26 апреля 2023 г., 16:19:16 


\title{
ОБ УСЛОВИЯХ ОБРАТИМОСТИ РАЗНОСТНЫХ ОПЕРАТОРОВ
}

\author{
(c) 2019 г. Л. Ю. КАБАНЦОВА
}

\begin{abstract}
АннотАция. Доказана обратимость разностных операторов второго порядка с постоянными операторными коэффициентами, действующих в банаховом пространстве двусторонних векторных последовательностей при условии их равномерной инъективности (в частности, левой обратимости) либо сюръективности (в частности, правой обратимости) либо фредгольмовости.
\end{abstract}

Ключевые слова: разностный оператор второго порядка, равномерная инъективность, сюръективность, фредгольмовость, спектр, обратимость.

\section{ON THE INVERTIBILITY CONDITIONS OF FINITE-DIFFERENCE OPERATORS}

\author{
(c) 2019 L. YU. KABANTSOVA
}

\begin{abstract}
The invertibility of second-order finite-difference operators with constant operator coefficients acting in the Banach space of two-sided vector sequences is proved under the condition of their uniform injectivity (in particular, left invertibility) or surjectivity (in particular, right invertibility) or Fredholm property.
\end{abstract}

Keywords and phrases: second-order finite-difference operator, uniform injectivity, surjectivity, Fredholm property, spectrum, invertibility.

AMS Subject Classification: 39A70, 47B39

1. Введение. Выяснение условий обратимости операторов на основе их более простых свойств, таких как равномерная инъективности или сюръективность, представляет теоретический и в ряде задач практический интерес. На первый взгляд кажется, что класс сюръективных операторов либо класс равномерно инъективных операторов шире класса обратимых операторов, однако это не всегда так. Исследованию данной проблемы и посвящена настоящая статья.

Пусть $\mathcal{X}$ - комплексное банахово пространство.

Определение 1.1. Замкнутый оператор $\mathcal{A}: D(\mathcal{A}) \subset \mathcal{X} \rightarrow \mathcal{X}$ назовем равномерно инбективным (см. [2]), если существует такая постоянная $c>0$, что $\|\mathcal{A} x\| \geqslant c\|x\|$ для любого вектора $x \in D(\mathcal{A})$.

Ясно, что каждый равномерно инъективный оператор инъективен. Если оператор $\mathcal{A}$ имеет левый обратный $\mathcal{B}_{l}$, принадлежащий банаховой алгебре End $\mathcal{X}$ линейных ограниченных операторов, т.е. $\mathcal{B}_{l} \mathcal{A} x=x, x \in D(\mathcal{A})$, то он является равномерно инъективным. Существование правого обратного $\mathcal{B}_{r} \in$ End $\mathcal{X}$ (т.е. $\operatorname{Im} \mathcal{B}_{r} \subset D(\mathcal{A})$ и $\mathcal{A B}_{r} x=x, x \in \mathcal{X}$ ) влечет сюръективность оператора $\mathcal{A}$.

Определение 1.2. Замкнутый оператор $\mathcal{A}: D(\mathcal{A}) \subset \mathcal{X} \rightarrow \mathcal{X}$ назовем фредгольмовым, если его образ $\operatorname{Im} \mathcal{A}$ замкнут (т.е. $\overline{\operatorname{Im} \mathcal{A}}=\operatorname{Im} \mathcal{A})$, ядро $\operatorname{Ker} \mathcal{A}$ конечномерно и коядро Coker $\mathcal{A}=Y / \operatorname{Im} \mathcal{A}$ также конечномерно. 
В случае, если оператор $\mathcal{A}$ имеет компактную резольвенту либо допускает представление $\mathcal{A}=$ $I+\mathcal{B}$, где $\mathcal{B}$ - компактный оператор, то инъективность оператора $\mathcal{A}$ либо его сюръективность влекут его обратимость (см. [4]). Однако, это утверждение неверно для общих операторов.

В настоящей статье исследуется проблема обратимости разностного оператора второго порядка с постоянными операторными коэффициентами, действующих в банаховом пространстве двусторонних векторных последовательностей, в условиях его равномерной инъективности, либо сюръективности, либо фредгольмовости. Основные результаты работы содержатся в трех теоремax 2.1, 2.2 и 2.3.

2. Основные результаты. Рассмотрим разностный оператор второго порядка $\mathfrak{D}_{2} \in$ End $l^{p}(\mathbb{Z}, \mathcal{X})$ вида

$$
\left(\mathfrak{D}_{2} x\right)(n)=x(n+2)+B_{1} x(n+1)+B_{2} x(n), \quad n \in \mathbb{Z}, \quad x \in l^{p},
$$

действующий в банаховом пространстве $l^{p}=l^{p}(\mathbb{Z}, \mathcal{X}), p \in[1, \infty]$, двусторонних последовательностей векторов из комплексного пространства $\mathcal{X}$, суммируемых со степенью $p \in[1, \infty)$ либо ограниченных в случае $p=\infty$. Будем считать, что $B_{1}, B_{2} \in \operatorname{End} \mathcal{X}$.

Разностному оператору $\mathfrak{D}_{2}$ соответствует характеристический многочлен (пучок операторов)

$$
H: \mathbb{C} \rightarrow \text { End } \mathcal{X}, \quad H(\lambda)=\lambda^{2} I+\lambda B_{1}+B_{2}, \quad \lambda \in \mathbb{C} .
$$

Всюду предполагается, что для спектра $\sigma(H)$ пучка $H$ выполнено условие

$$
\sigma(H) \cap \mathbb{T} \neq \mathbb{T},
$$

где $\mathbb{T}$ - окружность $\{\lambda \in \mathbb{C}:|\lambda|=1\}$. Таким образом, условие (2) означает, что на $\mathbb{T}$ имеются точки из резольвентного множества $\rho(H)$ пучка $H$.

Основными результатами статьи являются следующие три теоремы.

Теорема 2.1. Если разностный оператор $\mathfrak{D}_{2}: l^{p} \rightarrow l^{p}, p \in[1, \infty]$, равномерно инбективен, то он обратим.

Следствие 2.1. Если оператор $\mathfrak{D}_{2}: l^{p} \rightarrow l^{p}, p \in[1, \infty]$, обратим слева, то он обратим.

Теорема 2.2. Если разностный оператор $\mathfrak{D}_{2}: l^{p} \rightarrow l^{p}, p \in[1, \infty]$, сюрбективен, то он обраmuм.

Следствие 2.2. Обратимый справа оператор $\mathfrak{D}_{2}: l^{p} \rightarrow l^{p}, p \in[1, \infty]$, обратим.

Теорема 2.3. Если разностный оператор $\mathfrak{D}_{2}: l^{p} \rightarrow l^{p}, p \in[1, \infty]$, фредгольмов, то он обраmuм.

Замечание 2.1. Если не выполнено условие $(2)$ на спектр $\sigma(H)$ пучка $H$, то можно привести пример обратимого слева, но не обратимого разностного оператора.

3. Обратимость равномерно инъюктивных, сюръективных и фредгольмовых разностных операторов первого порядка. Изучению вопроса обратимости разностного оператора второго порядка при условии его равномерной инъективности либо сюръективности либо фредгольмовости предпошлем исследование данного вопроса для разностного оператора первого порядка.

Рассмотрим разностный оператор $\mathfrak{D} \in$ End $l^{p}(\mathbb{Z}, \mathcal{X})$ вида

$$
(\mathfrak{D} x)(n)=x(n+1)-B x(n), \quad n \in \mathbb{Z}, \quad x \in l^{p},
$$

действующий в банаховом пространстве $l^{p}=l^{p}(\mathbb{Z}, \mathcal{X}), p \in[1, \infty]$, двусторонних последовательностей векторов комплексного пространства $\mathcal{X}$, суммируемых со степенью $p \in[1, \infty)$ либо ограниченных в случае $p=\infty$. Для любой последовательности $x \in l^{p}$ положим

$$
\|x\|_{p}=\left(\sum_{k \in \mathbb{Z}}\|x(k)\|^{p}\right)^{1 / p}, \quad p \in[1, \infty), \quad\|x\|_{\infty}=\sup _{k \in \mathbb{Z}}\|x(k)\|, \quad p=\infty .
$$


Всюду предполагается, что оператор $B$ принадлежит банаховой алгебре End $\mathcal{X}$ и для спектра $\sigma(B)$ оператора $B$ выполнено условие

$$
\sigma(B) \cap \mathbb{T} \neq \mathbb{T}, \quad \mathbb{T}=\{\lambda \in \mathbb{C}:|\lambda|=1\} .
$$

Таким образом, условие $(3)$ означает, что на $\mathbb{T}$ имеются точки из резольвентного множества $\rho(B)$ оператора $B$.

При доказательстве основных утверждений (теорем) используется следующее утверждение (см. [7, теорема 4.5.7], а также [1]).

Теорема 3.1. Оператор $\mathfrak{D}: l^{p} \rightarrow l^{p}, p \in[1, \infty]$, обратим в точности тогда, когда выполнено условие

$$
\sigma(B) \cap \mathbb{T}=\varnothing
$$

m.е. спектр оператора $B$ не пересекается с единичной окружностью.

Теорема 3.2. Если разностный оператор $\mathfrak{D}: l^{p} \rightarrow l^{p}, p \in[1, \infty]$, равномерно ингективный, то он обратим.

Доказательство теоремъ 3.2. Пусть разностный оператор $\mathfrak{D} \in$ End $l^{p}$ равномерно инъективен. Известно (см. [6]), что из равномерной инъективности оператора $\mathfrak{D}$ в банаховом пространстве $l^{p}$ при некотором $p \in[1, \infty]$ следует его равномерная инъективность в банаховом пространстве $l^{q}$ при любом $q \in[1, \infty]$. Следовательно, без ограничения общности можно считать, что $p=\infty$.

Таким образом, существует такая постоянная $c>0$, что для любого $x \in l^{\infty}$ выполнено неравенство

$$
\|\mathfrak{D} x\|_{\infty} \geqslant c\|x\|_{\infty}
$$

Рассмотрим последовательность $x(n)=x_{0} \gamma^{n}$, где $x_{0} \in \mathcal{X},|\gamma|=1$, т.е. $\gamma \in \mathbb{T}, n \in \mathbb{Z}$. Применим оператор $\mathfrak{D}$ к данной последовательности:

$$
(\mathfrak{D} x)(n)=x_{0} \gamma^{n+1}-B x_{0} \gamma^{n}=\gamma^{n}\left(x_{0} \gamma I-B x_{0}\right), \quad n \in \mathbb{Z} .
$$

Согласно неравенству (4) имеет место оценка

т.е.

$$
\sup _{n \in \mathbb{Z}}\left\|\gamma^{n}(B-\gamma I) x_{0}\right\| \geqslant c\left\|x_{0}\right\|, \quad \gamma \in \mathbb{T},
$$

$$
\left\|(B-\gamma I) x_{0}\right\| \geqslant c\left\|x_{0}\right\|, \quad \gamma \in \mathbb{T} .
$$

Таким образом, операторы вида $B-\gamma I, \gamma \in \mathbb{T}$, равномерно инъективны с той же постоянной $c$.

Покажем, что на единичной окружности нет точек спектра оператора $B$. Предположим противное, т.е. что существует граничная точка $\gamma_{0}$ множества $\sigma(B) \cap \mathbb{T}$. В силу условия (3) в ее окрестности существует такая последовательность $\gamma_{n}$ чисел из $\rho(B)$, что $\gamma_{n} \in \mathbb{T}$ и при этом $\gamma_{0}=\lim _{n \rightarrow \infty} \gamma_{n}$

Так как $\gamma_{n} \in \rho(B)$, то справедливо неравенство

$$
\left\|\left(B-\gamma_{n} I\right) x_{0}\right\| \geqslant c\left\|x_{0}\right\| \quad \text { для любого вектора } x_{0} \in \mathcal{X} .
$$

Выберем $x_{0}=\left(B-\gamma_{n} I\right)^{-1} y_{0}$. Тогда для произвольного $y_{0} \in \mathcal{X}$ имеем

$$
\left\|\left(B-\gamma_{n} I\right)^{-1} y_{0}\right\| \leqslant \frac{1}{c}\left\|y_{0}\right\| .
$$

Представим оператор $B-\gamma_{0} I$ в виде

$$
B-\gamma_{0} I=\left(B-\gamma_{n} I\right)\left[I-\left(\gamma_{0}-\gamma_{n}\right)\left(B-\gamma_{n} I\right)^{-1}\right], \quad n \geqslant 1 .
$$

Введем обозначение

Согласно (5)

$$
B_{n}=\left(\gamma_{0}-\gamma_{n}\right)\left(B-\gamma_{n} I\right)^{-1}, \quad n \in \mathbb{Z}
$$

$$
\left\|B_{n}\right\|=\left|\gamma_{0}-\gamma_{n}\right|\left\|\left(B-\gamma_{n} I\right)^{-1}\right\| \leqslant\left|\gamma_{0}-\gamma_{n}\right| \frac{1}{c} \rightarrow 0 \quad \text { при } n \rightarrow \infty .
$$

Таким образом, существует такое число $n_{0} \in \mathbb{N}$, что для $n \geqslant n_{0}$ справедлива оценка $\left\|B_{n}\right\|<1$. Это означает, что оператор $I-B_{n}$ обратим. Поскольку оба сомножителя в формуле (6) обратимы, то 
обратим и оператор $B-\gamma_{0} I$. Это противоречит предположению, что $\gamma_{0} \in \sigma(B)$ и, следовательно, $\sigma(B) \cap \mathbb{T}=\varnothing$. Отсюда в силу теоремы 3.1 получаем, что оператор $\mathfrak{D}$ обратим. Теорема 3.2 доказана.

Следствие 3.1. Если оператор $\mathfrak{D}: l^{p} \rightarrow l^{p}, p \in[1, \infty]$, обратим слева, то он обратим.

Замечание 3.1. Если не выполнено условие (3) на спектр оператора $B$, то приводится пример обратимого слева, но не обратимого разностного оператора.

Пример 3.1. Пусть $X$ - гильбертово пространство, $U \in$ End $X$ - любая необратимая изометрия. Оператор $B$ определим следующим образом: $B=\beta U, \beta>2$. Поскольку оператор $U$ является изометрией, он равномерно инъективен. Оператор $U$ обратим слева, но при этом не является обратимым. Значит, оператор $B$ также имеет левый обратный $\left(B^{-1}\right)_{l}$, но обратимым не является.

Рассмотрим разностный оператор $\mathfrak{D} \in \operatorname{End} l^{\infty}(\mathbb{Z}, \mathcal{X})$ вида

$$
(\mathfrak{D} x)(n)=x(n+1)-B x(n), \quad n \in \mathbb{Z}, \quad x \in l^{\infty} .
$$

Покажем, что оператор $\mathfrak{D}$ равномерно инъективен. Рассмотрим последовательность $x_{0} \in l^{\infty}$, являющуюся решением разностного уравнения $\mathfrak{D} x_{0}=f$, где $f \in \operatorname{Im} \mathfrak{D}$. Пусть $n_{0} \in \mathbb{Z}$ - такое число, что $\left\|x_{0}\left(n_{0}\right)\right\| \geqslant \frac{1}{2}\left\|x_{0}\right\|_{\infty}$.

Запишем разностное соотношение для $n_{0} \in \mathbb{Z}$ :

$$
\left(\mathfrak{D} x_{0}\right)\left(n_{0}\right)=x_{0}\left(n_{0}+1\right)-B x_{0}\left(n_{0}\right)=f\left(n_{0}\right) .
$$

Отсюда $B x_{0}\left(n_{0}\right)=x_{0}\left(n_{0}+1\right)-f\left(n_{0}\right)$; учитывая, что $\beta>2$, получаем оценки

$$
\frac{\beta}{2}\left\|x_{0}\right\|_{\infty} \leqslant \beta\left\|x_{0}\left(n_{0}\right)\right\|=\left\|B x_{0}\left(n_{0}\right)\right\| \leqslant\left\|x_{0}\right\|_{\infty}+\|f\|_{\infty} .
$$

Таким образом, имеет место оценка

$$
\|f\|_{\infty}=\left\|\mathfrak{D} x_{0}\right\|_{\infty} \geqslant(\beta / 2-1)\left\|x_{0}\right\| .
$$

Так как $\beta>2$, оператор $\mathfrak{D}$ равномерно инъективен.

Однако спектр оператора $B$ не удовлетворяет условию (3), поскольку $\sigma(B)=\{\lambda \in \mathbb{C}:|\lambda| \leqslant \beta\}$ и, следовательно, $\rho(B) \cap \mathbb{T}=\varnothing$. Тогда в силу теоремы 3.1 приходим к выводу о необратимости разностного оператора $\mathfrak{D}$.

Покажем, что разностный оператор $\mathfrak{D} \in \operatorname{End} l^{\infty}$ является обратимым слева в банаховой алгебре End $l^{\infty}$ и один из его левых обратных операторов имеет вид

$$
\left(\left(\mathfrak{D}^{-1}\right)_{l} y\right)(n)=\sum_{m \in \mathbb{Z}} G_{0}(n-m) y(m-1), \quad y \in l^{\infty}(\mathbb{Z}, \mathcal{X}),
$$

где функция $G_{0}: \mathbb{Z} \rightarrow$ End $\mathcal{X}$ определяется формулой

$$
G_{0}(k)= \begin{cases}0, & k \geqslant 0, \\ -\left(B^{-1}\right)_{l}^{-k} I, & k \leqslant-1 .\end{cases}
$$

Для нашего примера проекторнозначные функции $P, Q: \mathbb{Z} \rightarrow$ End $\mathcal{X}$ определяются следующим образом: $P=P\left(\sigma_{\mathrm{int}}, B\right)=0, Q=I-P=I$, так как $\sigma_{\mathrm{int}}=\{\lambda \in \sigma(B):|\lambda|<1\}=\varnothing$.

Проверим, что оператор $\left(\mathfrak{D}^{-1}\right)_{l}$, задаваемый формулой $(7)$, является левым обратным для разностного оператора $\mathfrak{D} \in \operatorname{End} l^{\infty}$ :

$$
\begin{aligned}
\left(\left(\mathfrak{D}^{-1}\right)_{l} \mathfrak{D} x\right)(n)=- & \sum_{m=n+1}^{\infty}\left(B^{-1}\right)_{l}^{-n+m} x(m)+\sum_{m=n+1}^{\infty}\left(B^{-1}\right)_{l}^{-n+m} B x(m-1)= \\
= & -\sum_{m=n+1}^{\infty}\left(B^{-1}\right)_{l}^{-n+m} x(m)+\sum_{m=n+1}^{\infty}\left(B^{-1}\right)_{l}^{-n+m-1} x(m-1)=x(n), \quad n \in \mathbb{Z} .
\end{aligned}
$$

Теорема 3.3. Если разностный оператор $\mathfrak{D}: l^{p} \rightarrow l^{p}, p \in[1, \infty]$, сюргективен, то он обраmuм. 
Прежде чем перейти к доказательству теоремы 3.3, приведем ряд понятий, используемых при доказательстве.

Пусть $X, Y$ - банаховы пространства и $A: X \rightarrow Y$-линейный ограниченный оператор. Положим

$$
|A|_{+}=\inf \{\|A x\|:\|x\|=1\}, \quad|A|_{-}=\sup \left\{l \geqslant 0: l B_{Y} \subseteq A B_{X}\right\},
$$

где $B_{X}=\{x \in X:\|x\| \leqslant 1\}$ и $B_{Y}=\{y \in Y:\|y\| \leqslant 1\}$ - замкнутые единичные шары в $X$ и $Y$ соответственно. Очевидно, что $|A|_{+}$- наилучшая константа в неравенстве

$$
\|A x\| \geqslant|A|_{+}\|x\|, \quad x \in X .
$$

Таким образом,

$$
|A|_{+}=\sup \{l \geqslant 0:\|A x\| \geqslant l\|x\| \forall x \in X\} .
$$

В [7] эти константы $|A|_{+}$и $|A|_{-}$названы наименьшими нормами; в литературе их называют минимальным модулем и сюргективным модулем соответственно. Для наименьших норм справедливо следующее утверждение.

Теорема 3.4 (см. [7, теорема 1.3.2]). Пусть $A: X \rightarrow Y$-линейный ограниченный оператор. 1. $|A|_{+}>0$ тогда и только тогда, когда $\operatorname{Ker} A=\{0\}$ u $\operatorname{Im} A$ замкнут (т.е. оператор $A$ равномерно ингективен).

2. $|A|_{-}>0$ тогда и только тогда, когда $\operatorname{Im} A=Y$ (т.е. оператор А сюргективен).

3. Оператор A обратим тогда и только тогда, когда

$$
|A|_{+}=|A|_{-}=\frac{1}{\left\|A^{-1}\right\|}
$$

Доказательство теоремы 3.3. Напомним, что оператор $A$ и его сопряженный $A^{*}$ обратимы одновременно (см. [7, теорема 1.2.11]); при этом наименьшие нормы оператора $A \in \operatorname{End} X$ и сопряженного к нему оператора $A^{*} \in$ End $X^{*}$ удовлетворяют соотношениям

$$
\left|A^{*}\right|_{-}=|A|_{+}, \quad\left|A^{*}\right|_{+}=|A|_{-}
$$

(см. [7, утверждение 1.3.4]). Эти равенства и теорема 3.4 позволяют утверждать, что если оператор сюръективен, то сопряженный к нему равномерно инъективен.

Пусть $p \neq \infty$. Тогда сопряженным к пространству $l^{p}=l^{p}(\mathbb{Z}, \mathcal{X})$ является пространство $l^{p^{*}}=l^{p^{*}}\left(\mathbb{Z}, \mathcal{X}^{*}\right)$, где $p^{*}$ - сопряженный индекс, удовлетворяющий условию $1 / p+1 / p^{*}=1$ (см. например, [7, теорема 1.8.11], [5, гл. $4, \S 2])$. Покажем, что сопряженный к разностному оператору $\mathfrak{D}: l^{p} \rightarrow l^{p}, p \in[1, \infty)$, также является разностным оператором. Запишем заданный разностный оператор в виде

$$
(\mathfrak{D} x)(n)=(S-B) x(n), \quad n \in \mathbb{Z},
$$

где $S$-оператор сдвига последовательностей из $l^{p}, S \in \operatorname{End} l^{p}$, действующий по правилу $(S x)(n)=x(n+1), n \in \mathbb{Z}$. Имеют место равенства

$$
\mathfrak{D}^{*}=(S-B)^{*}=S^{*}-B^{*}, \quad n \in \mathbb{Z} .
$$

Оператор сдвига $S: l^{p} \rightarrow l^{p}, p \in[1, \infty)$, действующий в пространстве двусторонних векторных последовательностей, является унитарным при условии $p=2$, а $X$ - гильбертово пространство и, следовательно, $S^{*}=S^{-1}$, где оператор $S^{-1} \in \operatorname{End} l^{p^{*}}$ действует по правилу $\left(S^{-1} x\right)(n)=x(n-1)$. При всех остальных $p \leqslant \infty$ сопряженный к оператору сдвига задается также формулой $\left(S^{*} x\right)(n)=$ $x(n-1)$. Таким образом,

$$
\left(\mathfrak{D}^{*} x\right)(n)=x(n-1)-B^{*} x(n), \quad n \in \mathbb{Z} .
$$

Последнее соотношение позволяет утверждать, что $\mathfrak{D}^{*}$ - разностный оператор. Поскольку по условию теоремы 2.2 оператор $\mathfrak{D}$ сюръективен, то сопряженный к нему разностный оператор $\mathfrak{D}^{*}$ равномерно инъективен и по теореме 2.1 он обратим. Следовательно, обратим и исходный разностный оператор $\mathfrak{D}: l^{p} \rightarrow l^{p}, p \in[1, \infty)$.

Пусть $p=\infty$. В этом случае, как легко проверить, пространство $l^{1}=l^{1}\left(\mathbb{Z}, \mathcal{X}^{*}\right)$ является подпространством сопряженного $\left[l^{\infty}(\mathbb{Z}, \mathcal{X})\right]^{*}$ к $l^{\infty}(\mathbb{Z}, \mathcal{X})$, причем это подпространство инвариантно 
относительно сопряженного оператора $\mathfrak{D}^{*}:\left[l^{\infty}(\mathbb{Z}, \mathcal{X})\right]^{*} \rightarrow\left[l^{\infty}(\mathbb{Z}, \mathcal{X})\right]^{*}$. Поскольку, как отмечалось выше, оператор $\mathfrak{D}^{*}$ равномерно инъективен, его сужение на $l^{1}=l^{1}\left(\mathbb{Z}, \mathcal{X}^{*}\right)$ также равномерно инъективно. Но тогда по теореме 2.1 это сужение обратимо. Сопряженный к этому сужению оператор действует в $l^{\infty}\left(\mathbb{Z}, \mathcal{X}^{* *}\right)$ и является расширением первоначального оператора $\mathfrak{D}: l^{\infty}(\mathbb{Z}, \mathcal{X}) \rightarrow l^{\infty}(\mathbb{Z}, \mathcal{X})$. Значит, разностный оператор $\mathfrak{D}$ равномерно инъективен и, следовательно, обратим. Теорема 3.3 доказана.

Следствие 3.2. Обратимый справа оператор $\mathfrak{D}: l^{p} \rightarrow l^{p}, p \in[1, \infty]$, обратим.

Теорема 3.5. Если разностный оператор $\mathfrak{D}: l^{p} \rightarrow l^{p}, p \in[1, \infty]$, фредгольмов, то он обратим.

Доказательство теоремъ 3.5. Рассмотрим сначала случай $p \neq \infty$. Покажем, что ядро Ker $\mathfrak{D}$ фредгольмова разностного оператора $\mathfrak{D}: l^{p} \rightarrow l^{p}, p \in[1, \infty)$, состоит только из нуля. Предположим противное и рассмотрим последовательность $x_{0} \in l^{p}, p \in[1, \infty), x_{0} \neq 0$, принадлежащую $\operatorname{Ker} \mathfrak{D}$. Рассмотрим сдвиги последовательности $x_{0}$ на очень большие расстояния $\left(S\left(k_{i}\right) x_{0}\right)(n)=x_{0}\left(n+k_{i}\right), 1 \leqslant i \leqslant m, m \in \mathbb{N}$, где $k_{i}$ - достаточно большие числа. Заметим, что носители сдвигов supp $S\left(k_{i}\right) x_{0}, 1 \leqslant i \leqslant m$, почти не пересекаются (т.е. имеют конечное (возможно, нулевое) число общих элементов).

Покажем, что сдвиги $\left(S\left(k_{i}\right) x_{0}\right)(n)$ последовательности $x_{0}$ являются линейно независимыми функциями. Поскольку $p<\infty$, последовательность $x_{0}(n)$ стремится к нулю при $n \rightarrow \infty$ и, следовательно, в ней есть максимальный по модулю элемент. Без ограничения общности можно считать, что максимальным является элемент $x_{0}(0)$. Положим $x_{i}(n)=\left(S\left(-k_{i}\right) x_{0}\right)(n)=x_{0}\left(n-k_{i}\right)$, $i=1,2, \ldots, m$. Предположим противное, т.е. что последовательности $x_{1}, \ldots, x_{m}$ линейно зависимы. Это значит, что для некоторых коэффициентов $a_{i}, 1 \leqslant i \leqslant m$, удовлетворяющих условию $\left|a_{1}\right|+\left|a_{2}\right|+\cdots+\left|a_{m}\right| \neq 0$, справедливо соотношение

$$
a_{1} x_{1}+a_{2} x_{2}+\cdots+a_{m} x_{m}=0
$$

Выпишем эти равенства для координат $k_{i}$ :

$$
\begin{aligned}
a_{1} x_{1}\left(k_{1}\right)+a_{2} x_{2}\left(k_{1}\right)+\cdots+a_{m} x_{m}\left(k_{1}\right) & =0, \\
a_{1} x_{1}\left(k_{2}\right)+a_{2} x_{2}\left(k_{2}\right)+\cdots+a_{m} x_{m}\left(k_{2}\right) & =0, \\
\ldots \ldots \ldots+\cdots \cdots+\cdots \cdots+\cdots & \\
a_{1} x_{1}\left(k_{m}\right)+a_{2} x_{2}\left(k_{m}\right)+\cdots+a_{m} x_{m}\left(k_{m}\right) & =0 .
\end{aligned}
$$

Перепишем полученные равенства с учетом введенного обозначения $x_{i}(n)=x_{0}\left(n-k_{i}\right)$ :

$$
\begin{aligned}
& a_{1} x_{0}\left(k_{1}-k_{1}\right)+a_{2} x_{0}\left(k_{1}-k_{2}\right) \cdots+a_{m} x_{0}\left(k_{1}-k_{m}\right)=0, \\
& a_{1} x_{0}\left(k_{2}-k_{1}\right)+a_{2} x_{0}\left(k_{2}-k_{2}\right) \cdots+a_{m} x_{0}\left(k_{2}-k_{m}\right)=0,
\end{aligned}
$$

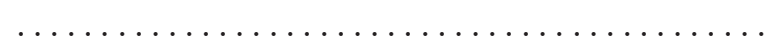

$$
\begin{aligned}
& a_{1} x_{0}\left(k_{m}-k_{1}\right)+a_{2} x_{0}\left(k_{m}-k_{2}\right) \cdots+a_{m} x_{0}\left(k_{m}-k_{m}\right)=0 .
\end{aligned}
$$

Окончательно получаем соотношения вида

$$
\begin{aligned}
& a_{1} x_{0}(0)+a_{2} x_{0}\left(k_{1}-k_{2}\right) \cdots+a_{m} x_{0}\left(k_{1}-k_{m}\right)=0, \\
& a_{1} x_{0}\left(k_{2}-k_{1}\right)+a_{2} x_{0}(0) \cdots+a_{m} x_{0}\left(k_{2}-k_{m}\right)=0 \text {, }
\end{aligned}
$$

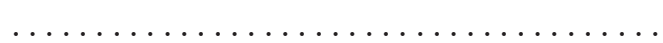

$$
\begin{aligned}
& a_{1} x_{0}\left(k_{m}-k_{1}\right)+a_{2} x_{0}\left(k_{m}-k_{2}\right) \cdots+a_{m} x_{0}(0)=0 .
\end{aligned}
$$

Будем смотреть на эти равенства как на систему относительно $a_{i}, i=1,2, \ldots, m$. В ней диагональные коэффициенты $x_{0}(0)$ большие, а остальные коэффициенты $x_{0}\left(k_{i}-k_{j}\right)$ можно сделать как угодно маленькими, подобрав числа $k_{i}$ таким образом, что $k_{i}-k_{j} \rightarrow \infty, i \neq j, i, j=1,2, \ldots, m$. Значит, можно добиться того, чтобы определитель этой системы был отличен от нуля. Но тогда все $a_{i}$ равны нулю, что противоречит сделанному предположению. А значит сдвиги последовательности $x_{0}$ на очень большие расстояния $\left(S\left(k_{i}\right) x_{0}\right)(n)=x_{0}\left(n+k_{i}\right), 1 \leqslant i \leqslant m, m \in \mathbb{N}$, являются линейно независимыми. 
Поскольку последовательность $x_{0}$ принадлежит $\operatorname{Ker} \mathfrak{D}$, то справедливо

$$
\mathfrak{D} x_{0}=0 .
$$

Применяя к этому равенству слева операторы сдвигов на большие расстояния, получаем

$$
S\left(k_{i}\right) \mathfrak{D} x_{0}=0, \quad 1 \leqslant i \leqslant m, m \in \mathbb{N} .
$$

Поскольку разностной оператор с постоянными коэффициентами перестановочен с операторами сдвигов, имеем

$$
\mathfrak{D}\left(S\left(k_{i}\right) x_{0}\right)=0, \quad 1 \leqslant i \leqslant m, m \in \mathbb{N} .
$$

Последнее соотношение означает, что все сдвиги $S\left(k_{i}\right) x_{0}, 1 \leqslant i \leqslant m$, исходной последовательности $x_{0}$ на очень большие расстояния попали в ядро разностного оператора $\mathfrak{D}$. Так как $m$ произвольно и с учетом линейной независимости сдвигов $S\left(k_{i}\right) x_{0}, 1 \leqslant i \leqslant m$, получаем, что ядро разностного оператора $\mathfrak{D}: l^{p} \rightarrow l^{p}, p \in[1, \infty)$, не является конечномерным, что противоречит условию теоремы. А значит, $\operatorname{Ker} \mathfrak{D}=\{0\}$, т.е. оператор $\mathfrak{D}$ инъективен.

По условию теоремы оператор $\mathfrak{D}$ фредгольмов, а значит образ оператора $\mathfrak{D}$ замкнут. В силу теоремы 3.4 [7, теорема 1.3.2] из замкнутости образа $\operatorname{Im} \mathfrak{D}$ разностного оператора и инъективности оператора $\mathfrak{D}$ следует, что $|\mathfrak{D}|_{+}>0$. Таким образом, существует константа $l=|\mathfrak{D}|_{+}>0$ такая, что

$$
\|\mathfrak{D} x\| \geqslant l\|x\| \quad x \in \mathcal{X}
$$

и, следовательно, разностный оператор $\mathfrak{D}: l^{p} \rightarrow l^{p}, p \in[1, \infty)$, равномерно инъективен. Отсюда, в силу теоремы 3.2 , оператор $\mathfrak{D}$ обратим.

Пусть $p=\infty$. В этом случае пространство $l^{1}=l^{1}\left(\mathbb{Z}, \mathcal{X}^{*}\right)$ является подпространством сопряженного $\left[l^{\infty}(\mathbb{Z}, \mathcal{X})\right]^{*}$ к $l^{\infty}(\mathbb{Z}, \mathcal{X})$, причем это подпространство инвариантно относительно сопряженного оператора $\mathfrak{D}^{*}:\left[l^{\infty}(\mathbb{Z}, \mathcal{X})\right]^{*} \rightarrow\left[l^{\infty}(\mathbb{Z}, \mathcal{X})\right]^{*}$. Поскольку оператор $\mathfrak{D}$ фредгольмов, $|\mathfrak{D}|_{-}>0$. Поэтому $\left|\mathfrak{D}^{*}\right|_{+}>0[7$, предположение 1.3 .4$]$, т.е. $\mathfrak{D}^{*}$ равномерно инъективен. Поскольку оператор $\mathfrak{D}^{*}$ равномерно инъективен, то его сужение на $l^{1}=l^{1}\left(\mathbb{Z}, \mathcal{X}^{*}\right)$ также равномерно инъективно. Но тогда по теореме 3.2 это сужение обратимо. Сопряженный к этому сужению действует в $l^{\infty}\left(\mathbb{Z}, \mathcal{X}^{* *}\right)$ и является расширением первоначального оператора $\mathfrak{D}: l^{\infty}(\mathbb{Z}, \mathcal{X}) \rightarrow l^{\infty}(\mathbb{Z}, \mathcal{X})$. Значит, разностный оператор $\mathfrak{D}$ равномерно инъективен и, следовательно, обратим. Теорема 3.5 доказана.

4. Доказательство теорем 2.1-2.3. Наряду с разностным оператором второго порядка (1) вида $\mathfrak{D}_{2}: l^{p}(\mathbb{Z}, \mathcal{X}) \rightarrow l^{p}(\mathbb{Z}, \mathcal{X})$ будем рассматривать разностный оператор первого порядка $\mathfrak{D}_{1}:$ $l^{p}\left(\mathbb{Z}, \mathcal{X}^{2}\right) \rightarrow l^{p}\left(\mathbb{Z}, \mathcal{X}^{2}\right)$, действующий по правилу

$$
\mathfrak{D}_{1}:\left(\begin{array}{l}
y_{1} \\
y_{2}
\end{array}\right) \longmapsto\left(\begin{array}{cc}
S & -I \\
B_{2} & S+B_{1}
\end{array}\right)\left(\begin{array}{l}
y_{1} \\
y_{2}
\end{array}\right), \quad\left(y_{1}, y_{2}\right) \in l^{p}\left(\mathbb{Z}, \mathcal{X}^{2}\right) .
$$

Здесь через $S$ обозначен оператор сдвига последовательностей из $l^{p}: S \in \operatorname{End} l^{p},(S x)(n)=x(n+1)$, $n \in \mathbb{Z}, x \in l^{p}$.

В [3] были получены результаты о совпадении ряда свойств введенных в рассмотрение операторов $\mathfrak{D}_{2}: l^{p}(\mathbb{Z}, \mathcal{X}) \rightarrow l^{p}(\mathbb{Z}, \mathcal{X})$ и разностного оператора $\mathfrak{D}_{1}: l^{p}\left(\mathbb{Z}, \mathcal{X}^{2}\right) \rightarrow l^{p}\left(\mathbb{Z}, \mathcal{X}^{2}\right)$. В частности, было установлено, что эти операторы одновременно обратимы, одновременно равномерно инъективны, одновременно сюръективны, одновременно фредгольмовы и т. д.

Теорема 2.1 непосредственно следует из теоремы 3.2 и теорем из [3] об одновременной равномерной инъективности и одновременной обратимости разностного оператора второго порядка $\mathfrak{D}_{2}: l^{p}(\mathbb{Z}, \mathcal{X}) \rightarrow l^{p}(\mathbb{Z}, \mathcal{X})$ и разностного оператора первого порядка $\mathfrak{D}_{1}: l^{p}\left(\mathbb{Z}, \mathcal{X}^{2}\right) \rightarrow l^{p}\left(\mathbb{Z}, \mathcal{X}^{2}\right)$

Теорема 2.2 непосредственно следует из теоремы 3.3 и теорем из [3] об одновременной сюръективности и одновременной обратимости разностного оператора второго порядка $\mathfrak{D}_{2}: l^{p}(\mathbb{Z}, \mathcal{X}) \rightarrow$ $l^{p}(\mathbb{Z}, \mathcal{X})$ и разностного оператора первого порядка $\mathfrak{D}_{1}: l^{p}\left(\mathbb{Z}, \mathcal{X}^{2}\right) \rightarrow l^{p}\left(\mathbb{Z}, \mathcal{X}^{2}\right)$.

Теорема 2.3 непосредственно следует из теоремы 3.5 и теорем из [3] об одновременной фредгольмовости и одновременной обратимости разностного оператора второго порядка $\mathfrak{D}_{2}: l^{p}(\mathbb{Z}, \mathcal{X}) \rightarrow$ $l^{p}(\mathbb{Z}, \mathcal{X})$ и разностного оператора первого порядка $\mathfrak{D}_{1}: l^{p}\left(\mathbb{Z}, \mathcal{X}^{2}\right) \rightarrow l^{p}\left(\mathbb{Z}, \mathcal{X}^{2}\right)$. 


\section{СПИСОК ЛИТЕРАТУРЫ}

1. Баскаков А. Г. Спектральный анализ оператора взвешенного сдвига с неограниченными операторными коэффициентами// Сиб. мат. ж. - 2001. - 42, № 6. - С. 1231-1243.

2. Баскаков А. Г. Спектральный анализ дифференциальных операторов с неограниченными операторными коэффициентами, разностные отношения и полугруппы разностных отношений// Изв. РАН. Сер. мат. - 2009. - 7, № 2. - С. 3-68.

3. Баскаков А.Г. Разностные операторы и операторные матрицы второго порядка// Изв. РАН. Сер. мат. - 2015. - 79, № 2. - С. 3-20.

4. Данфорд Н. Линейные операторы. Общая теория. - М.: ИЛ, 1962.

5. Колмогоров А. Н. Элементы теории функций и функционального анализа. - М.: Физматлит, 2004.

6. Aldloubi A., Baskakov A., Krishtal I. Slanted matrices, Banach frames and sampling// J. Funct. Anal. 2008. - 255. - P. 1667-1691.

7. Kurbatov V. G. Functional-Differential Operators and Equations. - Dordrecht: Kluwer Academic Publ., 1999.

Кабанцова Лариса Юрьевна

Воронежский государственный университет

E-mail: dlju@yandex.ru 